\title{
Kondo Breakdown and Hybridization Fluctuations in the Kondo-Heisenberg Lattice
}

\author{
I. Paul ${ }^{1,2}$, C. Pépin ${ }^{1}$ and M. R. Norman ${ }^{2}$ \\ ${ }^{1}$ SPhT, CEA-Saclay, L'Orme des Merisiers, 91191 Gif-sur-Yvette, France \\ ${ }^{2}$ Materials Science Division, Argonne National Laboratory, Argonne, IL 60439
}

(Dated: July 10, 2018)

\begin{abstract}
We study the deconfined quantum critical point of the Kondo-Heisenberg lattice in three dimensions using a fermionic representation for the localized spins. The mean-field phase diagram exhibits a zero temperature quantum critical point separating a spin liquid phase where the hybridization vanishes and a Kondo phase where it does not. Two solutions can be stabilized in the Kondo phase, namely a uniform hybridization when the band masses of the conduction electrons and the spinons have the same sign, and a modulated one when they have opposite sign. For the uniform case, we show that above a very small temperature scale, the critical fluctuations associated with the vanishing hybridization have dynamical exponent $z=3$, giving rise to a resistivity that has a $T \log T$ behavior. We also find that the specific heat coefficient diverges logarithmically in temperature, as observed in a number of heavy fermion metals.

PACS numbers: 71.27.+a, 72.15.Qm, 75.20.Hr, 75.30.Mb
\end{abstract}

A large number of experiments have been performed on metallic heavy fermion compounds close to a zero temperature phase transition (a quantum critical point (QCP)) driven by applied magnetic field, chemical doping or pressure [1]. In the quantum critical regime, the thermodynamics and transport properties are very unusual, violating the predictions of the Landau Fermi liquid theory of metals. The resistivity is quasi-linear in temperature over several decades, and in many cases the specific heat coefficient diverges logarithmically as the temperature is decreased. These unusual observations have motivated many theoretical studies that have attempted to capture these effects. Most theories [2, 3, 4, 5] are based on the assumption that at the QCP, the Fermi liquid is destabilized by spin density wave formation, and therefore the critical fluctuations are magnetic in nature. In $d=3$, these theories fail to capture simultaneously the linear temperature dependence of the resistivity and the divergence of the specific heat coefficient at low temperatures [ $[$ ]. More recently the problem has been approached from another perspective which takes the point of view that at the QCP, magnetic fluctuations suppress the formation of the heavy Fermi liquid, driving the effective Kondo temperature of the lattice $\left(T_{K}\right)$ to zero $[\underline{6}, \mathbf{7}, \underline{8}, 9]$. In this picture, the QCP is a bi-critical point where the metal experiences fluctuations due to the vanishing energy scale $T_{K}$ as well as the paramagnons. One feature that distinguishes between these two classes of theories is that the first predicts the Fermi volume to change smoothly across the QCP, while the second predicts an abrupt change [6].

In this Letter we explore the possibility that in the quantum critical regime, the unusual behavior in thermodynamics and transport is due to critical fluctuations, but of a non-magnetic order parameter associated with the vanishing energy scale $T_{K}$, and not due to paramagnons. The order parameter we advocate is the field $\sigma$ associated with the hybridization between the localized spins and the conduction electrons [10, 11]. At the QCP, the effective Kondo temperature for the lattice goes to zero, leading to a "Kondo breakdown" of the heavy Fermi liquid. The critical fluctuations of $\sigma$ are gapless excitations, and we study how these fluctuations influence the properties of the metal using the formalism of the large $N$ Kondo-Heisenberg model.

Beyond the mean-field level, the Kondo-Heisenberg model can be treated as a lattice gauge theory. Senthil et. al. [8] have examined the effect of the gauge fluctuations in this model, while Coleman et. al. [9] studied the zero temperature transport anomalies. In our work, we find a number of novel effects associated with the fluctuations of the $\sigma$ field which were not discovered in these earlier studies.

At the Kondo breakdown QCP where $\langle\sigma\rangle=0$, we observe two new phenomena: (1) $\sigma$ can order at a finite wavevector leading to spatial modulations of the Kondo hybridization analogous to the LOFF state of superconductivity [12]; (2) the presence of multiple energy scales, spread over a very large range in energy. The lowest scale is extremely small (of order $1 \mathrm{mK}$ ), above which, up to an ultraviolet cutoff of order the single ion Kondo temperature, the critical fluctuations of $\sigma$ exhibit a dynamical exponent $z=3$. This gives rise to a marginal Fermi liquid like behavior for the conduction electrons in $d=3$, with a resistivity that goes as $T \log T$. A logarithmic dependence is also found for the specific heat coefficient from both the gauge and $\sigma$ fluctuations.

Our starting point is the large $N$ formulation of the Kondo-Heisenberg model, where $N$ denotes the enlarged spin symmetry group $S U(N)$. It describes a broad band of conduction electrons interacting with localized spins through anti-ferromagnetic Kondo coupling $J_{K}>0$. The localized spins interact with each other via nearest neighbor exchange $J_{H}>0$. We work with a representation of the localized spins in terms of Abrikosov pseudo fermions $\vec{S}_{i}=\sum_{\alpha \beta} f_{i, \alpha}^{\dagger} \vec{\sigma}_{\alpha \beta} f_{i, \beta}$, where $(\alpha, \beta)=(1, N)$, with the 
constraint of $n_{f}=N / 2$ spinons per site $i$. The interactions which are quartic fermionic terms can be decoupled using Hubbard-Stratonovich fields $\varphi_{i j} \rightarrow \sum_{\alpha} f_{i \alpha}^{\dagger} f_{j \alpha}$ for the Heisenberg exchange, and $\sigma_{i}^{\dagger} \rightarrow \sum_{\alpha} f_{i \alpha}^{\dagger} c_{i \alpha}$ for the Kondo interaction. Following Ref. 8 we assume that in $d=3, \varphi$ condenses in a uniform spin liquid phase that gives dispersion to the spinons, which is an essential ingredient for Kondo breakdown to occur (physically we interpret the uniform spin liquid as a mean field description of the short range magnetic correlations that persist when a magnetic ground state is destroyed by quantum fluctuations). This gives the Lagrangian

$$
\begin{aligned}
\mathcal{L} & =\sum_{\langle i j\rangle \alpha}\left[c_{i \alpha}^{\dagger}\left(\partial_{\tau}+t_{i j}\right) c_{j \alpha}+f_{i \alpha}^{\dagger}\left(\partial_{\tau}+\varphi_{0} e^{i a_{i j}}+\lambda_{i} \delta_{i j}\right) f_{j \alpha}\right] \\
& -\frac{N}{2} \sum_{i} \lambda_{i}+\frac{N}{J_{K}} \sum_{i} \sigma_{i}^{\dagger} \sigma_{i}+\frac{N \varphi_{o}^{2}}{J_{H}} \\
& +\sum_{i \alpha}\left(c_{i \alpha}^{\dagger} f_{i \alpha} \sigma_{i}+\text { h.c. }\right)
\end{aligned}
$$

( $V$, the volume of the system, is set to 1 ). The above Lagrangian has a local $U(1)$ gauge invariance [13]. The Lagrange multiplier $\lambda_{i}$ (scalar potential) enforces the constraint $n_{f}=N / 2$ per site. Given a state which satisfies the above constraint, a single hop of a spinon will violate it. Consequently only simultaneous opposite hops of spinons between two neighboring sites are physically allowed. This implies that the local spinon current operator $J_{f i}=0$ at each site. The gauge fields $a_{i j}$ (vector potential) associated with the phase of $\varphi_{i j}$ ensure that this condition is satisfied.

There are two important parameters in Eq. 1 First, $\alpha=\varphi_{0} / D$, which is the ratio of the spinon to the conduction electron bandwidth $D$ (note from Eq. I that for $\sigma=0, \phi_{0}=J_{H}$ ). Second, while the spinon band is half-filled due to the constraint (henceforth we assume $N=2$ ), the conduction band filling is generic. Without any loss of generality we take the conduction band to be less than half filled. This implies that the Fermi wavevector of the spinon band, $k_{F 0}$, is different from that of the conduction band, $k_{F}$. We denote the mismatch by $q^{*}=k_{F 0}-k_{F}$. In the following we take $\alpha$ and $\left(q^{*} / k_{F}\right)$ to be small. We identify $\alpha D$ with the single ion Kondo scale $\left(T_{K}^{0}=D e^{-1 / \rho_{0} J_{K}}\right)$ which is typically $10 \mathrm{~K}$ in heavy fermions. Assuming $D \sim 10^{4} \mathrm{~K}$, we get $\alpha \sim 10^{-3}$.

At the mean-field level, the parameters $\varphi_{0},\left\langle\lambda_{i}\right\rangle$ and $\left\langle\sigma_{i}\right\rangle$ are determined by minimizing the free energy, $F$. The mean-field phase transition between the spin liquid state, $\left\langle\sigma_{i}\right\rangle=0$, and the heavy Fermi liquid state with a lattice Kondo temperature $T_{K} \approx \pi \rho_{0}\left\langle\sigma_{i}\right\rangle^{2}$, occurs when

$$
\frac{\partial^{2} F}{\partial\left|\sigma_{q}\right|^{2}}=\frac{1}{J_{K}}+\Pi_{f c}(q, 0)=0
$$

where $\Pi_{f c}(q, 0)$ is the static electron-spinon (fc) polarization. We solve this equation for two different
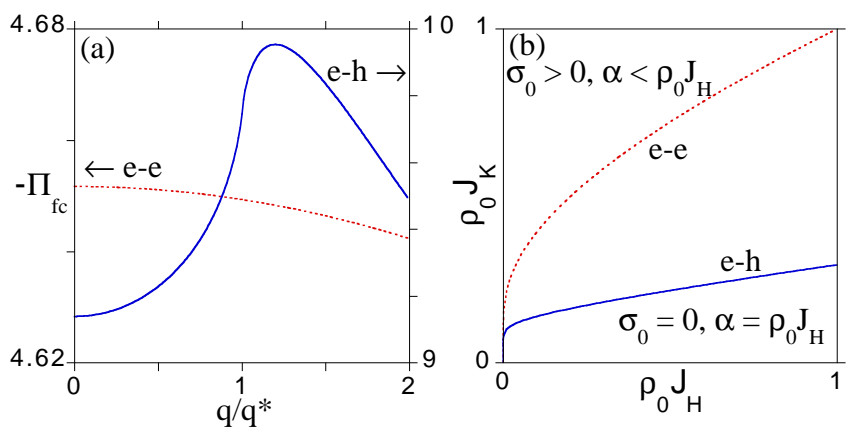

FIG. 1: (a) q dependence of $\Pi_{f c}\left(\alpha=0.01\right.$ and $\left.q^{*} / k_{F}=0.1\right)$. Note differing scales for the e-e and e-h cases. (b) Quantum critical point as a function of $J_{K}$ and $J_{H}$ for the e-e $(\mathrm{q}=0)$ and e-h $\left(\mathrm{q}=1.2 \mathrm{q}^{*}\right)$ cases.

situations, the result of which is depicted in Fig. 1. (i) e-e case, where both the bands are taken to be electron-like. Linearizing the fermionic dispersions we have $\epsilon_{k}=v_{F}\left(k-k_{F}\right)$ for the conduction electrons, and $\epsilon_{k}^{0}=\alpha v_{F}\left(k-k_{F}-q^{*}\right)$ for the spinons (where $k=|\mathbf{k}|$ ). For linearized dispersions, $\Pi_{f c}(q, 0)$ turns out to be $q$ independent. Inclusion of the curvature stabilizes a second order phase transition around $q=0$, the polarization taking the form $\Pi_{f c}(q, 0)=\frac{\rho_{0}}{1-\alpha}\left(\ln \alpha+\frac{1-\alpha^{2}+2 \alpha \ln \alpha}{4(1-\alpha)^{2}} \frac{q^{2}}{k_{F}^{2}}\right)$ where $\rho_{0}=1 / D$ is the conduction electron density of states at the Fermi energy. In this case the $T=0$ phase transition occurs at a critical Kondo coupling of $J_{K}^{c}=1 /\left(\rho_{0} \ln \left(1 / \rho_{0} J_{H}\right)\right)$ [14]. (ii) e-h case, where the conduction band is taken to be electron-like as before, while the spinon band is hole-like with a linearized dispersion $\epsilon_{k}^{0}=-\alpha v_{F}\left(k-k_{F}-q^{*}\right)$. In this case we find $\Pi_{f c}(q, 0)=\frac{\rho_{0}}{1+\alpha}\left(\ln \frac{\alpha v_{F}^{2}\left|q^{* 2}-q^{2}\right|}{D^{2}(1+\alpha)^{2}}-2+\frac{q^{*}}{q} \ln \frac{q^{*}+q}{\left|q^{*}-q\right|}\right)$, which has a minimum at $q=1.2 q^{*}$ independent of $\alpha$. In this state $T_{K}$ is modulated, with nodes in space where $T_{K}$ vanishes. This solution is similar to the spin density wave instability encountered in chromium [15] and in the LOFF state of superconductivity [12, 16]. Fig. 1a shows that for parabolic bands the minimum of the effective potential is lower in the e-h case than in the e-e case. Thus, for parabolic bands, the modulated solution is more stable (Fig. 1b). However the question of which solution is realized in real compounds will be material dependent.

We now turn to the fluctuations around the meanfield solution. We present our results for the simpler e-e case, leaving the more complex e-h case for a later paper. In the quantum critical regime there are two important types of gapless fluctuations, namely the gauge fluctuations associated with $a_{i j}$ and the critical fluctuations of the Kondo bosons $\sigma$.

The gauge fluctuations of this theory have been studied earlier by Senthil et. al. [8]. Here we summarize the salient points to put our work in perspective. It is convenient to work in the Coulomb gauge $\vec{\nabla} \cdot \vec{a}=0$, where the vector gauge fields $a_{\mu}(\mu=x, y, z)$ are purely transverse 

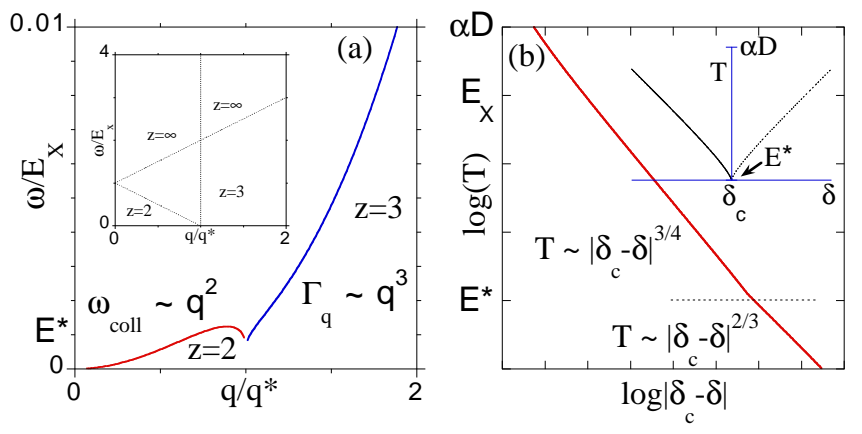

FIG. 2: (a) Structure of $D_{\sigma}$ for the e-e case $(\alpha<<1)$ at the QCP for positive frequencies. $z$ is the dynamical exponent in the various regions, which are delineated by the dashed lines, equal to $\alpha v_{F}\left(q^{*} \pm q\right)$ and $v_{F}\left(q^{*} \pm q\right)$. $\omega_{\text {coll }}$ is the zero of $D_{\sigma}^{-1}$ in the $\mathrm{z}=2$ regime (a propagating mode), and $\Gamma_{q}$ is the dispersion of the damped mode in the $\mathrm{z}=3$ regime (maximum of the imaginary part of $D_{\sigma}$ ). Note the presence of energy scales $E^{*} \sim 10^{-4} \alpha D$ and $E_{X}=\alpha v_{F} q^{*} \sim 10^{-1} \alpha D$. (b) Phase diagram for the e-e case $(\alpha<<1)$. Note the crossover from $\mathrm{z}=2$ to $\mathrm{z}=3$ behavior at $E^{*}$ (dotted line). The inset shows the phase diagram on a linear scale. The solid line is the crossover line in the Kondo phase, the dashed line the crossover line in the spin liquid phase.

[13. The fluctuations of $\lambda$ decouple from those of $a_{\mu}$, and give rise to a screened Coulomb interaction. As such, they are massive and can be neglected. The gauge fields $a_{\mu}$, which act as vectorial Lagrange multipliers to ensure that the local spinon current $J_{f i}=0$, do not have any intrinsic dynamics of their own. Their dynamics is generated via coupling with the spinon band, and therefore they are over-damped. For frequencies smaller than the spinon bandwidth $\alpha D$, the transverse gauge field propagator $D_{\mu \nu}(\mathbf{x}, \tau)=\left\langle T_{\tau}\left[a_{\mu}(\mathbf{x}, \tau) a_{\nu}(0,0)\right]\right\rangle$ has the standard form $D_{\mu \nu}\left(q, i \Omega_{n}\right)=\left(\delta_{\mu \nu}-q_{\mu} q_{\nu} / q^{2}\right) \Pi^{-1}\left(q, i \Omega_{n}\right)$, with $\Pi\left(q, i \Omega_{n}\right) \propto\left[\left(q / 2 k_{F 0}\right)^{2}+\left|\Omega_{n}\right| /\left(\alpha v_{F} q\right)\right]$. This is the typical form for excitations with dynamical exponent $z=$ 3 , which in $d=3$ are known [17] to give a contribution to the specific heat coefficient $\gamma \equiv-\partial^{2} F / \partial T^{2} \propto \ln (\alpha D / T)$ and to the static spin susceptibility $\delta \chi_{s} \propto T^{2} \ln (\alpha D / T)$. Finally, when the compact nature of the $U(1)$ gauge group on the lattice is taken into account, the gauge fluctuations convert the finite temperature mean-field transition line into a crossover line [8, 18].

In the quantum critical regime, the fluctuations of the complex order parameter fields $\left(\sigma_{i}^{\dagger}, \sigma_{i}\right)$ are massless as well (ignoring for the moment the $T$-dependent mass generated by the quartic $|\sigma|^{4}$ coupling). The propagator for these fluctuations is defined as $D_{\sigma}(x, \tau)=$ $\left\langle T_{\tau}\left[\sigma^{\dagger}(x, \tau) \sigma(0,0)\right]\right\rangle$ with $D_{\sigma}^{-1}\left(q, i \Omega_{n}\right)=1 / J_{K}+$ $\Pi_{f c}(q, 0)+\Delta \Pi_{f c}\left(q, i \Omega_{n}\right)$ where

$$
\Delta \Pi_{f c}\left(q, i \Omega_{n}\right)=\sum_{ \pm} \frac{\rho_{0}\left[\mp X_{1 \pm} \ln \left(X_{1 \pm}\right) \pm X_{2 \pm} \ln \left(X_{2 \pm}\right)\right]}{2 \alpha v_{F} q(1-\alpha)}
$$

with $X_{1 \pm}=-\alpha i \Omega_{n} \pm \alpha v_{F} q-\alpha v_{F} q^{*}, X_{2 \pm}=-i \Omega_{n} \pm$ $\alpha v_{F} q-\alpha v_{F} q^{*}$, and $\Pi_{f c}(0,0)=\rho_{0} \ln (\alpha) /(1-\alpha)\left(\Delta \Pi_{f c}\right.$ is the dynamical part of the fc polarization). The different regimes of $D_{\sigma}\left(q, i \Omega_{n}\right)$ with their associated dynamical exponents $z$ are summarized in Fig. 2a. At high energies, one finds $z=\infty$ behavior consistent with quasi-local behavior. But we find physical properties are dominated by the $z=3$ and $z=2$ regimes. These two regimes can be understood as follows. Due to the mismatch between the two Fermi surfaces, a minimum momentum is necessary to excite inter-band (fc) particle-hole pairs. As a result for $\Omega<\alpha v_{F}\left(q^{*}-q\right)$, excitations of $\sigma$ do not decay into particle-hole pairs but propagate ballistically with $D_{\sigma}^{-1}\left(q, i \Omega_{n}\right) \approx \rho_{0}\left[q^{2} /\left(4 k_{F}^{2}\right)-i \Omega_{n} / E_{X}\right]$, i.e., $z=2$ (where $\left.E_{X}=\alpha v_{F} q^{*}\right)$. This behavior is cutoff for frequencies $\Omega>E^{*}$ with

$$
E^{*}=c \alpha D\left(q^{*} / k_{F}\right)^{3} \quad \text { and } c \sim 10^{-1},
$$

above which the dynamical exponent $z$ changes from 2 to 3 . For most of the phase space, the spectrum for the fluctuations of $\sigma$ lie within the inter-band particlehole continuum, making their dynamics over-damped with $D_{\sigma}^{-1}\left(q, i \Omega_{n}\right) \approx \rho_{0}\left[q^{2} /\left(4 k_{F}^{2}\right)+\pi\left|\Omega_{n}\right| /\left(2 \alpha v_{F} q\right)\right]$, i.e., $z=3$. The energy scale in the $\mathrm{z}=3$ regime is $\Gamma_{q} / E_{X} \approx$ $q^{3} /\left(2 \pi k_{F}^{2} q^{*}\right)$ which has an infrared cutoff at $E^{*}$ because of the mismatch vector $q^{*}$. The ultraviolet cutoff scale for the $z=3$ regime is $\alpha v_{F}\left(q+q^{*}\right)$ which is of order $\alpha D$ for $q \sim k_{F}$. The energies $E^{*}$ (infrared) and $\alpha D$ (ultraviolet) appear as crossover scales for any physical property that is affected by the excitations of $\sigma$. For a one impurity Kondo scale $\alpha D \sim 10 K$, and $q^{*} / k_{F} \sim 10^{-1}$ [19], we estimate $E^{*} \sim 1 m K$. $E^{*}$ is therefore a very small energy scale which is essentially unobservable.

The crossover lines in $T$ that define the quantum critical region are symmetric around the QCP $\delta=\delta_{c}$, where $\delta=1 /\left(\rho_{0} J_{K}\right)$ (Fig. $2 \mathrm{~b}$ ). These are determined by the $T$ dependent mass generated by the quartic $|\sigma|^{4}$ coupling. For $T<E^{*}$, we find that the leading contribution is from the $z=2$ regime (proportional to $T^{3 / 2}$ for $d=3$ ), so that the crossover temperature $T \propto\left|\delta-\delta_{c}\right|^{2 / 3}$, while for $T>E^{*}$ the $z=3$ regime dominates giving a crossover temperature $T \propto\left|\delta-\delta_{c}\right|^{3 / 4}$ for $d=3$.

Next we examine the contribution to the free energy from the fluctuations of $\sigma$. We find that the leading contribution is entirely due to the $z=3$ regime since it comes from a much larger phase space volume (the $z=2$ contribution is similar to that of a gapless magnon mode). For $E^{*}<T<\alpha D$, we find $F(T) \sim \int n_{B} \operatorname{Im} \ln \left(D_{\sigma}^{-1}\right) \sim$ $-k_{F}^{3} T^{2} /(9 \alpha D) \ln (\alpha D / T)$, which is a typical result for $z=3$ excitations. This implies a contribution to the specific heat coefficient $\gamma \sim 2 k_{F}^{3} /(9 \alpha D) \ln (\alpha D / T)$, which adds to a similar contribution from the transverse gauge fluctuations. For $T<E^{*}$, the infrared cutoff sets in, and the specific heat coefficient from the $\sigma$ fluctuations saturates. This regime is then dominated by the logarithmic contribution from the transverse gauge fields [17]. 
We now calculate the self energy of the conduction electrons due to the hybridization fluctuations $(c \rightleftharpoons f+\sigma)$. This is defined as $\Sigma_{c}\left(k, i \omega_{n}\right)=T \sum_{\omega_{n}, q} G_{f}\left(k+q, i \omega_{n}+\right.$ $\left.i \Omega_{n}\right) D_{\sigma}\left(q, i \Omega_{n}\right)$, where $G_{f}^{-1}\left(k, i \omega_{n}\right)=\left(i \omega_{n}-\epsilon_{k}^{0}\right)$ is the inverse propagator of the spinons. As in the case of the free energy, we find that the leading contribution is due to the $z=3$ regime of $D_{\sigma}\left(q, i \Omega_{n}\right)$. At $T=0$ and for $E^{*}<\omega<\alpha D$, we find $\operatorname{Im} \Sigma_{c}\left(k_{F}, \omega\right) \sim k_{F}^{2} /\left(6 \pi \alpha v_{F} \rho_{0}\right) \omega$. The temperature dependence of $\operatorname{Im} \Sigma_{c}\left(k_{F}, \omega=0, T\right)$ involves a frequency integral weighted by the factor $n_{B}+n_{F}=1 / \sinh (\Omega / T)$. This makes the integral logarithmically divergent in the infrared, which is cutoff by $E^{*}$. For $E^{*}<T<\alpha D$ we find

$$
\operatorname{Im} \Sigma_{c}\left(k_{F}, \omega=0, T\right) \sim k_{F}^{2} /\left(6 \pi \alpha v_{F} \rho_{0}\right) T \ln \left(2 T / E^{*}\right) .
$$

For $\omega, T<E^{*}$, the self energy is Fermi liquid like.

We turn to the $T$-dependence of the static spin susceptibility, $\chi_{s}(T)$. At the mean field level, we find what is usual for band fermions, namely a constant part plus a $T^{2}$ term. To calculate the correction beyond mean field $\left(\delta \chi_{s}\right)$ due to the Kondo bosons, we note that in a magnetic field $B$, there is an additional $(B /(\alpha D))^{2}$ contribution to $D_{\sigma}^{-1}\left(q, i \Omega_{n}\right)$. This gives $\delta \chi_{s}(T) \propto T^{4 / 3}$ for $E^{*}<T<\alpha D$, and a $T^{2}$ dependence below $E^{*}$ (so below $E^{*}$ the $T^{2} \ln (T)$ contribution due to the gauge bosons dominates).

Finally we discuss the temperature dependence of the resistivity, $\rho$, that is obtained in the quantum critical regime. Eq. 5 gives the $T$-dependence of the inverse lifetime $\tau_{c}^{-1} \propto \operatorname{Im} \Sigma_{c}(T)$ of the conduction electrons. For one band models experiencing $q \simeq 0$ scattering, this lifetime cannot be associated with the transport lifetime, $\tau_{\text {tr }}$, because the leading contribution to the self energy comes from forward scattering processes which are not effective in relaxing the current. Consequently, when vertex corrections are taken into account, $\tau_{\mathrm{tr}}^{-1}$ acquires an additional temperature dependence proportional to $q^{2} \sim T^{2 / z}$. However, our model consists of two bands, one of light particles (the conduction electrons) which scatter from very heavy particles (the spinons) 20]. As such, the charge neutral spinons act as an effective bath for the relaxation of the conduction electron current (the other charge carrying modes, the complex $\sigma$ bosons, have over-damped dynamics, and therefore the current is mostly carried by the conduction electrons). The first non-zero vertex correction involves two $\sigma$ boson exchange processes. Such a correction is small by a factor of $\alpha$. Therefore $\tau_{\operatorname{tr}}$ can be identified with $\tau_{c}$, and for $E^{*}<T<\alpha D$ we find

$$
\delta \rho(T) \equiv \rho(T)-\rho(0) \propto T \ln \left(2 T / E^{*}\right) .
$$

For $T<E^{*}, \delta \rho(T) \propto T^{2}$, but $E^{*}$ is extremely small $(\sim 1 \mathrm{mK})$. Thus, the Kondo-Heisenberg model captures one of the most mysterious features of quantum criticality in heavy fermion compounds, namely the quasi-linear resistivity observed for most compounds over a large temperature range.

In conclusion, we studied the Kondo breakdown QCP of the Kondo-Heisenberg model in $d=3$. Over a large temperature range, we find that the critical fluctuations have a dynamical exponent $z=3$, giving rise to marginal Fermi liquid behavior for the conduction electrons. The specific heat coefficient has a $\log (1 / T)$ dependence, while the resistivity has a $T \log T$ behavior. The Kondo-Heisenberg model is characterized by multiple energy scales, and as such shows great promise in explaining the various subtleties associated with heavy fermion quantum critical behavior.

We thank the hospitality of the KITP where this work was initiated. We also acknowledge J. Schmalian, P. Sharma, and A. Chubukov for extensive discussions. This work was supported by the U.S. Dept. of Energy, Office of Science, under Contract No. W-31-109-ENG-38 and in part by the National Science Foundation under Grant No. PHY99-07949.

[1] G. Stewart, Rev. Mod. Phys. 73, 797 (2001).

[2] J. A. Hertz, Phys. Rev. B 14, 1165 (1976).

[3] A. J. Millis, Phys. Rev. B 48, 7183 (1993).

[4] T. Moriya and T. Takimoto, J. Phys. Soc. Japan 64, 960 (1995).

[5] A. Rosch, A. Schroder, O. Stockert and H. von Lohneysen, Phys. Rev. Lett. 79, 159 (1997); A. Rosch, ibid 82, 4280 (1999).

[6] P. Coleman, C. Pepin, Q. Si and R. Ramazashvili, J. Phys.: Condens. Matter 13, R723 (2001).

[7] Q. Si, S. Rabello, K. Ingersent and J. L. Smith, Nature 413, 804 (2001); D. R. Grempel and Q. Si, Phys. Rev. Lett. 91, 026401 (2003); P. Sun and G. Kotliar, Phys. Rev. Lett. 91, 037209 (2003).

[8] T. Senthil, S. Sachdev and M. Vojta, Phys. Rev. Lett. 90, 216403 (2003); T. Senthil, M. Vojta and S. Sachdev, Phys. Rev. B 69, 035111 (2004).

[9] P. Coleman, J. B. Marston and A. J. Schofield, Phys. Rev. B 72, 245111 (2005).

[10] N. Read and D. M. Newns, J. Phys. C 16, 3273 (1983); N. Read, J. Phys. C 18, 2651 (1985).

[11] A. J. Millis and P. A Lee, Phys. Rev. B 35, 3394 (1987).

[12] P. Fulde and R. A. Ferrell, Phys. Rev. 135, A550 (1964); A. I. Larkin and Y. N. Ovchinnikov, Sov. Phys. JETP 20, 762 (1965).

[13] L. B. Ioffe and A. I. Larkin, Phys. Rev. B 39, 8988 (1989); P. A. Lee and N. Nagaosa, Phys. Rev. B 46, 5621 (1992).

[14] That is, $\alpha D=D e^{-1 / \rho_{0} J_{K}^{c}}=T_{K}^{0}$.

[15] T. M. Rice, Phys. Rev. B 2, 3619 (1970).

[16] For spherical Fermi surfaces, as many ordering wave vectors as allowed by lattice symmetry will condense, each with a modulus of $q$.

[17] T. Holstein, R. E. Norton and P. Pincus, Phys. Rev. B 8, 2649 (1973); M. Yu. Reizer, ibid 40, 11571 (1989).

[18] N. Nagaosa and P. A. Lee, Phys. Rev. B 61, 9166 (2000).

[19] Heavy fermion metals have complex Fermi surfaces where 
both conduction and $\mathrm{f}$ surfaces are large. As a consequence, large values of $q^{*}$ are unlikely. Very small values of $q^{*}$ are possible due to degeneracies. Within our simple model, a value of $q^{*} / k_{F} \sim 0.1$ is a reasonable estimate.

[20] In a simple Boltzman approximation for f-c scattering, the factor weighting the transport would be $1-\alpha \cos (\theta) \simeq$ 1, see J. M. Ziman, Electrons and Phonons (Oxford Univ. Pr., London, 1960), p. 376-377. 